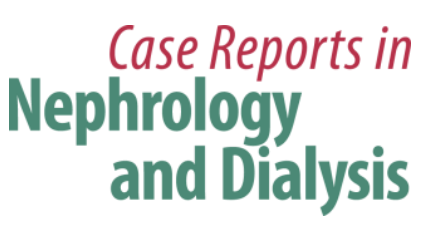

Case Rep Nephrol Dial 2019;9:85-91

DOI: $10.1159 / 000501260$

Published online: June 26, 2019

(C) 2019 The Author(s)

Published by S. Karger AG, Basel

www.karger.com/cnd

This article is licensed under the Creative Commons Attribution-NonCommercial 4.0 International License (CC BY-NC) (http://www.karger.com/Services/OpenAccessLicense). Usage and distribution for commercial purposes requires written permission.

\title{
PLA2R-Negative Membranous Nephropathy Presenting as a Prodrome of IgG4-Related Autoimmune Pancreatitis
}

\author{
Magdalini Velegraki $^{a} \quad K$ ostas G. Stylianou ${ }^{b}$ Dimitrios Xydakis $^{c}$ \\ Hariklia Gakiopoulou $^{d} \quad$ Evangelos Voudoukis $^{a} \quad$ Dimitra Lygerou $^{b}$ \\ Eleftheria-Kleio Dermitzaki ${ }^{b}$ Aphrodite Mpitouli ${ }^{a}$ Gregorios Paspatis $^{a}$ \\ Angeliki Theodoropoulou ${ }^{a}$ \\ aDepartment of Gastroenterology, Venizeleion Hospital, Heraklion, Greece; ${ }^{b}$ Department \\ of Nephrology, University Hospital of Heraklion, Heraklion, Greece; 'Department of

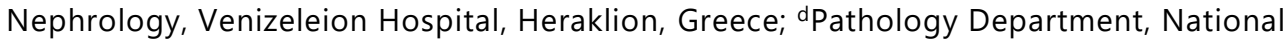 \\ and Kapodistrian University of Athens, Athens, Greece
}

\section{Keywords}

Autoimmune pancreatitis · Membranous nephropathy · PLA2R · Diabetes mellitus · IgG4-related disease · Endoscopic ultrasound

\begin{abstract}
Membranous nephropathy (MN) with tubulointerstitial nephritis (TIN) is an established manifestation of immunoglobulin G4 (IgG4)-related disease (IgG4-RD). A pathological feature aiming to distinguish between primary and secondary $\mathrm{MN}$ is the presence or absence of glomerular staining for phospholipase A2 receptor (PLA2R), respectively. Isolated MN without TIN has been rarely reported in the context of IgG4-RD. This case report describes a patient with a history of MN successfully treated with steroids and cyclophosphamide, who, 3 years later, presented with unexplained exacerbation of diabetes mellitus due to IgG4-related autoimmune pancreatitis. Pancreatitis was treated, and diabetes improved after treatment with steroids. Based on the presence of isolated IgG4 glomerular capillary deposits along with negative staining for PLA2R and the metachronous appearance of autoimmune pancreatitis, MN was
\end{abstract}




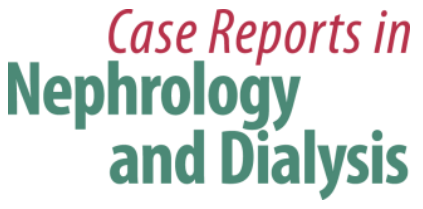

Case Rep Nephrol Dial 2019;9:85-91

DOI: $10.1159 / 000501260$

(c) 2019 The Author(s). Published by S. Karger AG, Basel www.karger.com/cnd

Velegraki et al.: PLA2R-Negative Membranous Nephropathy Presenting as a Prodrome of IgG4-Related Autoimmune Pancreatitis

retrospectively classified as secondary to IgG4-RD. Isolated IgG4-positive/PLA2R-negative MN without TIN can be a prodrome of IgG4-RD, reminiscent of MN secondary to neoplasms.

(C) 2019 The Author(s)

Published by S. Karger AG, Basel

\section{Introduction}

Immunoglobulin G4 (IgG4)-related disease (IgG4-RD) is an immune-mediated condition comprised of several disorders that can involve one or multiple organs and appears with particular histopathologic, serologic, and clinical features $[1,2]$. The affected organs include the pancreas, gall bladder, liver, thyroid, lungs, kidneys, skin, cranial nerves, retroperitoneum, prostate, pituitary, and salivary and lacrimal glands. The hallmarks of IgG4-RD are swelling and enlargement of the affected organ(s) with characteristic lymphoplasmacytic infiltrations composed of IgG4+ plasma cells, storiform fibrosis, obliterative phlebitis, mild to moderate eosinophilia, and remarkably high serum IgG and IgG4 [3].

Autoimmune pancreatitis (AIP) is a rare disorder of autoimmune etiology, which is associated with characteristic clinical, histologic, and morphologic findings [4]. Type 1 AIP is the prototypical form of IgG4-RD and can be associated with involvement of multiple organs [5, 6]. The most common symptoms are obstructive jaundice, abdominal pain, and weight loss. New-onset diabetes mellitus (DM) may also occur. Type 2 AIP is a primary pancreatic disorder without relation to IgG4 [7].

Kidneys involvement with tubulointerstitial nephritis (TIN), as part of the IgG4-RD, was first described in 2004 [8]. It is characterized by interstitial infiltration of IgG4-positive plasma cells, IgG deposits along the tubular basement membranes, and fibrosis [9, 10]. IgG4related membranous nephropathy (MN) is much less common than IgG4-related TIN, and these 2 pathologies usually occur together [11].

Here, we report a patient who presented with deregulation of preexisting type $2 \mathrm{DM}$ as the first manifestation of AIP. The patient's medical history included "idiopathic" MN 3 years earlier, which was finally attributed to IgG4-RD after being diagnosed with type 1 AIP.

\section{Case Presentation}

A 70-year-old male was admitted to our hospital due to abdominal pain localized in the epigastrium, weight loss of $10 \mathrm{~kg}$ over the previous 2 months, and deregulation of preexisting type 2 DM that necessitated more aggressive antidiabetic therapy with insulin during the preceding 6 months. He had a history of MN successfully treated with methylprednisolone and cyclophosphamide 3 years ago, hypertension, dyslipidemia, and benign prostate hyperplasia. Physical examination revealed only epigastric tenderness. Laboratory tests showed an elevated erythrocyte sedimentation rate of $45 \mathrm{~mm} / 1 \mathrm{~h}$ (normal range [NR] 0-20 mm/1 h), normochromic, normocytic anemia (hemoglobin at $12.3 \mathrm{~g} / \mathrm{dL}$, NR $13.4-17.4 \mathrm{~g} / \mathrm{dL}$ ) and increased glycated hemoglobin (HbA1c) at 8\%. Hepatic and pancreatic enzymes, arterial $\mathrm{pH}$, and kidney function were all normal. A contrast-enhanced abdominal computed tomography (CT) revealed enlargement and edema of the pancreatic body and tail along with decreased density of the pancreatic parenchyma and inflammation of the peripancreatic fat tissue; findings compatible with interstitial pancreatitis. A significant narrowing of the pancreatic duct at the head of the pancreas was also noted. A magnetic resonance imaging (MRI) of the abdomen and an MR cholangiopancreatography confirmed the diagnosis of pancreatitis and excluded 


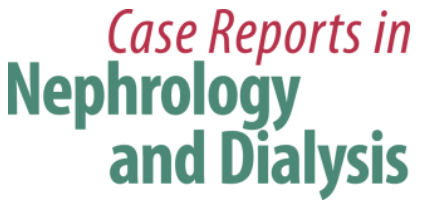

Case Rep Nephrol Dial 2019;9:85-91

DOI: $10.1159 / 000501260$

(c) 2019 The Author(s). Published by S. Karger AG, Basel www.karger.com/cnd

Velegraki et al.: PLA2R-Negative Membranous Nephropathy Presenting as a Prodrome of IgG4-Related Autoimmune Pancreatitis

the presence of gallstones or choledocholithiasis. Notably, the above-mentioned findings were not present in an abdominal CT performed 3 years ago when MN was diagnosed. The patient was assessed with endoscopic ultrasound (EUS) which revealed findings suggestive of chronic pancreatitis with 1 major B and 3 minor features, based upon the Rosemont classification [12]. More specifically, there was a diffusely enlarged pancreatic gland with poor echotexture, lobularity with "honeycombing," stranding, hyperechoic foci without shadowing, and a normal main pancreatic duct with dilated side branches. A fine-needle aspiration biopsy was performed. Cytological examination demonstrated low-grade dysplasia of the ductal epithelium with no evidence of malignancy. The patient underwent a second EUS examination, and new tissue specimens were obtained for histological examination. Immunohistochemistry revealed lymphoplasmocytic infiltration of the gland with IgG4-positive plasmocytes. Further serological findings showed increased serum IgG4 levels $(1,220 \mathrm{mg} / \mathrm{dL}, \mathrm{NR} 8-140 \mathrm{mg} / \mathrm{dL})$, while antinuclear antibodies and rheumatoid factor were not present. Based on the revised HISORT (Histology, Imaging, Serology, other Organ involvement and Response to Therapy) criteria [5], the patient was diagnosed with type 1 AIP, and oral prednisolone treatment was initiated at a dose of $40 \mathrm{mg}$ daily for 4 weeks followed by a tapering of $5 \mathrm{mg}$ per week. Two weeks later, the abdominal pain had subsided, and fasting blood glucose levels had decreased. Ten weeks after initiation of prednisolone, the abnormal CT findings had significantly improved and serum IgG4 levels had decreased at $394 \mathrm{mg} / \mathrm{dL}$. Moreover, HbA1c declined at $6.5 \%$, insulin was withheld, and oral antidiabetic drugs were restarted.

The renal biopsy was reevaluated, and immunohistochemistry to detect IgG/IgG4 and phospholipase A2 receptor (PLA2R) was performed. Immunohistochemistry demonstrated a strong, diffuse, and global staining for both IgG and IgG4 along the walls of the glomerular capillaries with a fine granular pattern (Fig. 1a-c), whereas PLA2R was negative (Fig. 1d). The interstitial tissue did not show infiltration by lymphocytes, plasmocytes, or any other inflammatory cells (Fig. 1e), and so, no staining for IgG or IgG4 was observed in the interstitium (Fig. 1f). No staining along the tubular basement membranes was found (Fig. 1f). Of note, no morphological signs of secondary membranous glomerulopathy, for instance increased mesangial cellularity, were observed (Fig. 1g, h). A serum sample frozen at the time of kidney biopsy was retrospectively examined for the presence of anti-PLAR antibodies by the immunofluorescence method (Euroimmun AG, Germany) and tested negative. Despite the presence of unselective proteinuria at the time of serum sampling, IgG4 levels were slightly high (at 186 $\mathrm{mg} / \mathrm{dL}$ ), implying the presence of IgG4-RD from the outset.

\section{Discussion}

IgG4-RD is a novel immune-mediated condition that can present with multi-organ involvement. IgG4-RD has also been observed in several subjects previously diagnosed with conditions that were regarded as organ-specific entities. We report a patient who presented with exacerbation of DM as the first manifestation of AIP. According to the literature, DM is present in $70 \%$ of all type 1 AIP cases [13]. Importantly, AIP-induced DM improves with corticosteroid treatment [14]. Corticosteroids recover pancreatic endocrine function by suppression of lymphoplasmacytic infiltration and cytokine production [14]. A unique feature of the presented case is that our patient had formerly been diagnosed with $\mathrm{MN}$ as a separate, organspecific entity since there was no evidence of extrarenal manifestations suggestive of IgG4-RD at that time. Three years later, the diagnosis of type 1 AIP led to the diagnosis of IgG4-RD, raising the possibility that the earlier glomerulopathy had been part of the systemic disease. 


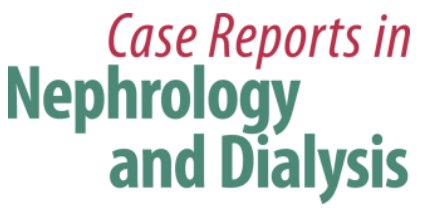

The association between IgG4-RD and MN is not well established. The most common renal manifestation of IgG4-RD is plasma cell-rich TIN with "storiform" fibrosis. In 2013, Alexander et al. [15] demonstrated that MN can be part of IgG4-related kidney disease in 7\% of patients, usually in association with concurrent TIN. Given that IgG4 is the main subtype observed in the immunodeposits of primary MN, it is quite difficult to connect a MN with an underlying IgG4-RD, if there is no appropriate clinical history suggesting an IgG4-RD, especially in the absence of TIN. In secondary forms of MN, such IgG4 antibodies are absent or less prevalent [16], with the exception (by definition) of MN in the context of IgG4-RD. When IgG4-RD is diagnosed upon other organ(s) involvement, then suspicion should be raised that MN might be part of this syndrome, as rare cases of primary MN have been described in the context of IgG4RD [17-20]. The differential diagnosis between primary and secondary MN in a patient with known IgG4-RD is mainly based on the presence or absence of other markers, such as PLA2R. In our case, IgG4 was positive in the immune deposits, as expected either in a primary MN or in a MN secondary to IgG4-RD. However, the retrospectively performed PLA2R analysis was negative (both anti-PLAR antibodies in serum and PLAR antigen in glomeruli), indicating a secondary etiology and, in this case, a MN in the context of an IgG4-RD. Another interesting point in our case is that the diagnosis of IgG4-RD was established after the diagnosis of MN, reminiscent of cases where MN can precede the discovery of a neoplastic disease. The time frame for the appearance of the manifestations of IgG4-RD from different organs can be very wide. For instance, a patient reported by Cravedi et al. [19] with IgG4-related MN was diagnosed 3 years after AIP, similar to our patient. So, in cases of IgG4-positive/PLA2R-negative MN associated with other IgG4-RD manifestations, the possibility of a MN in the context of IgG4-RD should be kept in mind to guarantee the subsequent appropriate investigation and follow-up of the patients.

\section{Conclusion}

We bring this case to the attention of physicians for 2 reasons. First, our case suggests that type 1 AIP should always be considered as part of the differential diagnosis in patients presenting with unexplained exacerbation of DM and a thorough workup should be performed in order to identify an existing pancreatic disorder. Secondly, isolated IgG4-positive/PLA2R-negative MN without TIN, although rare, can be a prodrome of IgG4-RD, reminiscent of MN secondary to neoplasms.

\section{Statement of Ethics}

A written consent for publication has been obtained from the patient, and all research was conducted ethically in accordance with the World Medical Association Declaration of Helsinki.

\section{Disclosure Statement}

The authors declare that they have no competing interests. 


\section{Case Reports in Nephrology and Dialysis}

Velegraki et al: PLA2R-Negative Membranous Nephropathy Presenting as a Prodrome of IgG4-Related Autoimmune Pancreatitis

\section{Funding Sources}

This work was not supported by any funding sources.

\section{Author Contributions}

M.V., E.V., and A.T. analyzed and interpreted the patient data regarding the pancreatic disease and D.X. regarding the kidney disease. H.G. performed the histological examination of the kidney and was a major contributor in writing the manuscript. M.V., D.L., E.-K.D., and K.S. wrote the manuscript. A.T. and G.P. supervised and approved the final version to be published. All authors read and approved the final manuscript. The data that support the findings of this study are available from the corresponding author upon reasonable request.

\section{References}

1 Fragoulis GE, Moutsopoulos HM. IgG4 syndrome: old disease, new perspective. J Rheumatol. 2010 Jul;37(7):1369-70.

2 Mahajan VS, Mattoo H, Deshpande V, Pillai SS, Stone JH. IgG4-related disease. Annu Rev Pathol. 2014;9(1):315-47.

3 Deshpande V, Zen Y, Chan JK, Yi EE, Sato Y, Yoshino T, et al. Consensus statement on the pathology of IgG4related disease. Mod Pathol. 2012 Sep;25(9):1181-92.

$4 \quad$ Finkelberg DL, Sahani D, Deshpande V, Brugge WR. Autoimmune pancreatitis. N Engl J Med. 2006 Dec;355(25):2670-6.

5 Chari ST, Takahashi N, Levy MJ, Smyrk TC, Clain JE, Pearson RK, et al. A diagnostic strategy to distinguish autoimmune pancreatitis from pancreatic cancer. Clin Gastroenterol Hepatol. 2009 Oct;7(10):1097-103.

6 Cornell LD, Chicano SL, Deshpande V, Collins AB, Selig MK, Lauwers GY, et al. Pseudotumors due to IgG4 immune-complex tubulointerstitial nephritis associated with autoimmune pancreatocentric disease. Am J Surg Pathol. 2007 Oct;31(10):1586-97.

7 Kanno A, Masamune A, Okazaki K, Kamisawa T, Kawa S, Nishimori I, et al.; Research Committee of Intractable Diseases of the Pancreas. Nationwide epidemiological survey of autoimmune pancreatitis in Japan in 2011. Pancreas. 2015 May;44(4):535-9.

8 Takeda S, Haratake J, Kasai T, Takaeda C, Takazakura E. IgG4-associated idiopathic tubulointerstitial nephritis complicating autoimmune pancreatitis. Nephrol Dial Transplant. 2004 Feb;19(2):474-6.

9 Cortazar FB, Stone JH. IgG4-related disease and the kidney. Nat Rev Nephrol. 2015 Oct;11(10):599-609.

10 Saeki T, Kawano M, Mizushima I, Yamamoto M, Wada Y, Nakashima H, et al. The clinical course of patients with IgG4-related kidney disease. Kidney Int. 2013 Oct;84(4):826-33.

11 Stylianou K, Maragkaki E, Tzanakakis M, Stratakis S, Gakiopoulou H, Daphnis E. Acute Interstitial Nephritis and Membranous Nephropathy in the Context of IgG4-Related Disease. Case Rep Nephrol Dial. 2014 Dec;5(1):44-8.

12 Catalano MF, Sahai A, Levy M, Romagnuolo J, Wiersema M, Brugge W, et al. EUS-based criteria for the diagnosis of chronic pancreatitis: the Rosemont classification. Gastrointest Endosc. 2009 Jun;69(7):1251-61

13 Okazaki K, Kawa S, Kamisawa T, Ito T, Inui K, Irie H, et al.; Working Committee of the Japan Pancreas Society and the Research Committee for Intractable Pancreatic Disease supported by the Ministry of Health, Labour and Welfare of Japan. Amendment of the Japanese Consensus Guidelines for Autoimmune Pancreatitis, 2013 I. Concept and diagnosis of autoimmune pancreatitis. J Gastroenterol. 2014 Apr;49(4):567-88.

14 Kamisawa T, Okazaki K, Kawa S, Ito T, Inui K, Irie H, et al.; Working Committee of the Japan Pancreas Society and the Research Committee for Intractable Pancreatic Disease supported by the Ministry of Health, Labour and Welfare of Japan. Amendment of the Japanese Consensus Guidelines for Autoimmune Pancreatitis, 2013 III. Treatment and prognosis of autoimmune pancreatitis. J Gastroenterol. 2014 Jun;49(6):961-70.

15 Alexander MP, Larsen CP, Gibson IW, Nasr SH, Sethi S, Fidler ME, et al. Membranous glomerulonephritis is a manifestation of IgG4-related disease. Kidney Int. 2013 Mar;83(3):455-62.

16 Huang CC, Lehman A, Albawardi A, Satoskar A, Brodsky S, Nadasdy G, et al. IgG subclass staining in renal biopsies with membranous glomerulonephritis indicates subclass switch during disease progression. Mod Pathol. 2013 Jun;26(6):799-805. 
17 Acevedo Ribó M, Ahijado Hormigos FJ, Diaz F, Romero Molina M, Fernandez Rojo MA, Garcia Rubiales MA, et al. IgG4-related disease and idiopathic membranous nephropathy: "The clothes do not make the man". Clin Nephrol. 2016 Dec;86(12):345-48.

18 Sueta S, Kondo M, Matsubara T, Yasuhara Y, Akiyama S, Imai E, et al. Membranous nephropathy associated with type 1 autoimmune pancreatitis and dominant glomerular IgG4 deposit. CEN Case Rep. 2014 May;3(1):18-23.

19 Cravedi P, Abbate M, Gagliardini E, Galbusera M, Buelli S, Sabadini E, et al. Membranous nephropathy associated with IgG4-related disease. Am J Kidney Dis. 2011 Aug;58(2):272-5.

20 Palmisano A, Corradi D, Carnevali ML, Alberici F, Silini EM, Gatti R, et al. Chronic periaortitis associated with membranous nephropathy: clues to common pathogenetic mechanisms. Clin Nephrol. 2010 Dec;74(6):48590. 


\section{Case Reports in Nephrology and Dialysis}

Case Rep Nephrol Dial 2019;9:85-91

DOI: 10.1159/000501260 (c) 2019 The Author(s).
www.karger.com/cnd

Velegraki et al: PLA2R-Negative Membranous Nephropathy Presenting as a Prodrome of IgG4-Related Autoimmune Pancreatitis

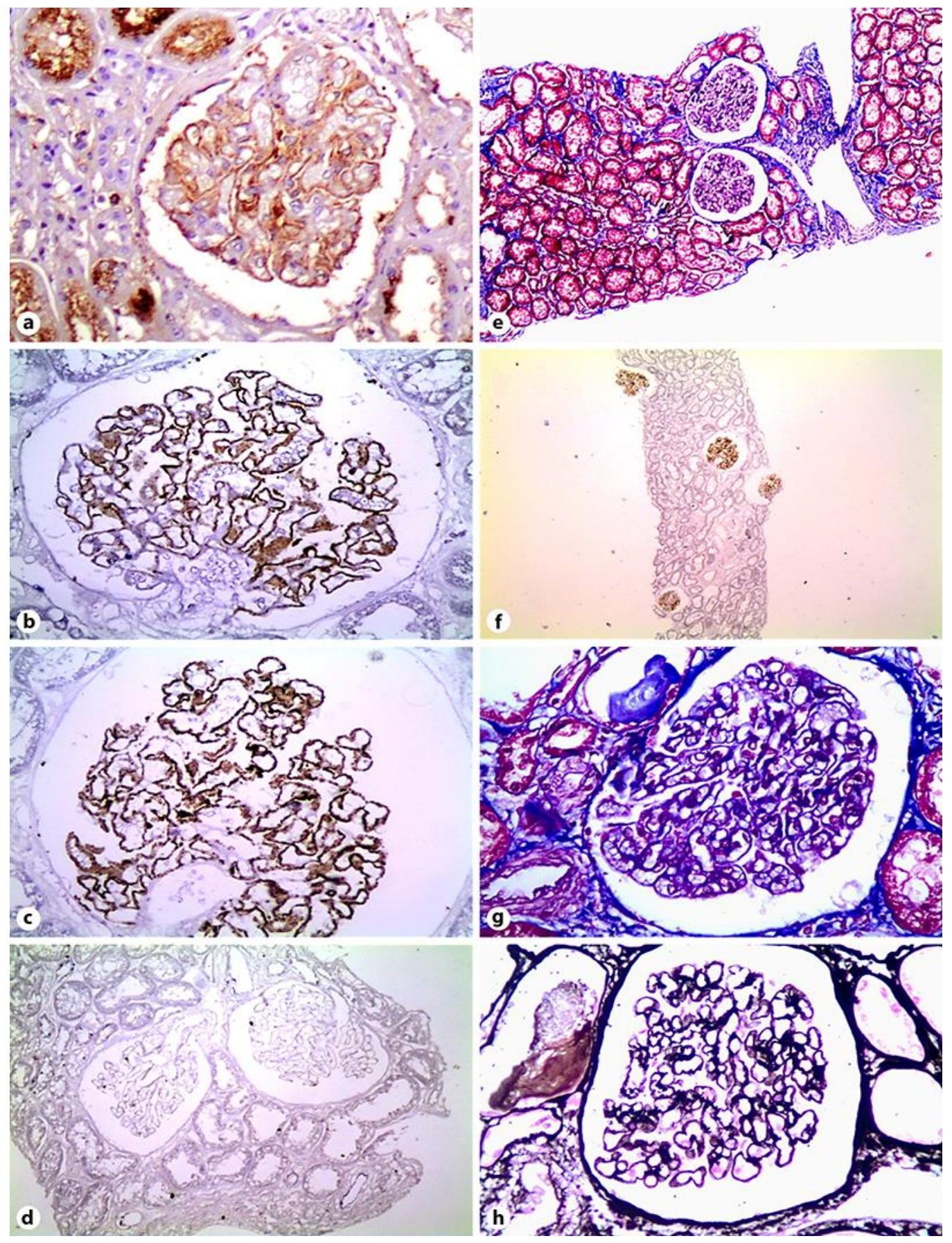

Fig. 1. Kidney biopsy findings. a Strong global granular IgG staining along the capillary walls; IgG, $\times 400$. b, c Strong global fine granular IgG4 staining along the capillary walls; IgG4, $\times 400$. d Negative PLA2R immunohistochemical staining; $\times 200$. e Membranous nephropathy without tubulointerstitial nephritis; Masson, $\times 100$. $f$ Low-power magnification $(\times 40)$ showing diffuse IgG4 glomerular staining with no staining in the interstitium. $\mathbf{g}$ Membranous nephropathy without morphological signs of secondary etiology (without mesangial cellularity); Masson, $\times 400$. $\mathbf{h}$ Membranous nephropathy without morphological signs of secondary etiology (without mesangial cellularity); Silver, $\times 400$. 